\title{
African Rice Cultivation. Wissens- und Technologietransfer von westafrikanischem Reisanbau nach South Carolina, USA
}

\author{
Jasmin Joppich \\ Kerngebiet: Wirtschafts- und Sozialgeschichte \\ eingereicht bei: Ass.-Prof. Dr. Ute Hasenöhrl \\ eingereicht im: SoSe 2018 \\ Rubrik: SE-Arbeit
}

\begin{abstract}
African Rice Cultivation. Knowledge and technology transfer from West Africa to South Carolina, USA

The following paper is about the knowledge and technologies of rice cultivation that enslaved Africans brought from West Africa to colonial South Carolina. The paper examines why and in what ways West African technologies of rice cultivation were used and adapted in South Carolina to maximise production and profits, how rice production evolved after the Civil War in 1865, and whether there were any further developments in US rice cultivation.
\end{abstract}

\section{Einleitung}

„Rice is the world's single most important staple food; small wonder, then, that the rice industry plays a leading role in feeding the world population. Rice processing is still carried out in a wide variety of ways, from the small village mills which are fed by hand and run at a capacity of a few 100 kilos an hour, to highly sophisticated mills that run at 100 tons or more an hour [...]."1

Im Jahr 2017 wurden weltweit 756,7 Millionen Tonnen Reis produziert. Obwohl sich die USA mit einer Produktionsmenge von 8,1 Millionen Tonnen aufPlatz 13 der Liste der füh-

Joachim Sontag, Introduction, in: Joachim Sontag (Hrsg.), Rice processing. The comprehensive guide to global technology and innovative products, Clenze 2014, S. 3-4, hier S. 4. 
renden Reisproduktionsländer befinden, gehören sie dennoch zu den fünf führenden Reisexportländern:2

"[The] United States is annually one of the top five rice exporting countries as U.S. production far exceeds domestic demand for rice. The United States currently exports roughly $40 \%$ of the rice it produces [...]."3

Auch in der Saison 2016/2017 exportierten die USA etwa vierzig Prozent des in den USA angebauten und vorrätigen Reises. Im Vergleich dazu führte Vietnam knapp ein Fünftel aus, während dies Indien, Marktführer im Reisexport, mit nur etwa neun Prozent des Gesamtangebots tat. ${ }^{4}$ Das Angebot überstieg die heimische Nachfrage nach Reis dementsprechend weit mehr als in Indien oder Vietnam. Historisch gesehen war der Reisanbau in Nordamerika von weitaus größerer Bedeutung als heute. Eine wichtige Anbauregion mit langer Kultivierungstradition war South Carolina. ${ }^{5}$ Die Reisindustrie erlebte dort ab den 1690er-Jahren einen rasanten Aufschwung, ${ }^{6}$ wobei dieser Boom bis in die 1970er-Jahre als alleiniger Verdienst europäischer Siedler*innen wahrgenommen und deren Einfallsreichtum zugeschrieben wurde. Diese einseitige, eurozentristische Perspektive änderte sich spätestens mit Peter Woods Monographie „Black Majority", die im Jahr 1974 erschien,, und in der Wood folgendermaßen argumentierte: „Particular know-how, rather than lack of it, was one factor that made black labor attractive to English colonialists."

Neben Peter Wood beschäftigten sich mit der Geschichte der Reisindustrie und der Frage nach der Agency der versklavten Afrikaner*innen in South Carolina und Georgia insbesondere auch Daniel Littlefield, der u. a. das Werk „Rice and Slaves. Ethnicity and the Slave Trade in Colonial South Carolina"9 verfasste, und Judith Carney, die zahlreiche Essays zur Reisgeschichte in den USA veröffentlichte und ebenfalls eine Monographie („Black Rice. The African Origins of Rice Cultivation in the Americas“) schrieb, die im Jahr 2001 erschien. Für ihre Dissertation verbrachte sie einige Monate in Gambia, forschte in Archiven und arbeitete in den Reisfeldern. ${ }^{10}$ Carney argumentiert, dass versklavte Menschen aus den westafrikanischen Reisanbaugebieten über ein umfangreiches und ganzheitliches Wissens- sowie Technologiesystem über den Reisanbau und die -verar-

2 Food and Agriculture Organization of the United Nations, FAO Rice Market Monitor (RMM) (1), 2018, S. 31-33, [http://www.fao.org/3/19243EN/i9243en.pdf], eingesehen 7. 2. 2019.

3 Terry J. Siebenmorgen, Rice Production, Consumption and Milling Capacity in the United States of America, in: Sontag (Hrsg.), Rice processing, S. 206-213, hier S. 207.

$4 \quad$ Food and Agriculture Organization of the United Nations, FAO Rice Market Monitor (RMM), S. 35.

5 Judith Carney, Out of Africa. Colonial Rice History in the Black Atlantic, in: Sandra G. Harding (Hrsg.), The postcolonial science and technology studies reader, Durham, NC 2011, S. 140-149, hier S. 140.

6 Judith Carney, From Hands to Tutors. African Expertise in the South Carolina Rice Economy, in: Agricultural History 67 (1993), Nr. 3, S. 1-30, hier S. 1, [https://www.jstor.org/stable/3744227], eingesehen 27. 7. 2018.

7 Carney, Out of Africa, S. 141

8 Peter Wood, Black Majority, zit. n. Judith Carney, Black Rice. The African Origins of Rice Cultivation in the Americas, Cambridge 2001, S. 69.

9 Daniel Littlefield, Rice and Slaves. Ethnicity and the Slave Trade in Colonial South Carolina, Baton Rouge, London 1981.

10 Wood, Black Majority, S. xi-xii. 
beitung verfügten und damit zum wirtschaftlichen Erfolg der einschlägigen Industrie in South Carolina und Georgia beitrugen. ${ }^{11}$

Die Ansätze von Wood, Littlefield und Carney sind bekannt als „black-rice-hypothesis“,12 bekannt Aspekte der Black-Rice-Hypothese wurden u. a. von Walter Hawthorne in Frage gestellt. Zwar kommt auch er zum Ergebnis, dass die Reisarbeiten in Westafrika und den USA größtenteils die gleichen gewesen wären, betont aber, dass sich die Einstellungen dazu wesentlich voneinander unterschieden hätten. Damit meint er die Differenz der Wahrnehmung von Arbeit von einerseits selbstständigen Bauernfamilien im vorkolonialen Westafrika und andererseits versklavten Zwangsarbeiter*innen in South Carolina und Georgia ebenso wie die Produktionsweisen der zwei Gruppen Subsistenzund Massenproduktion. ${ }^{13}$ Allerdings wies Carney bereits im Jahr 1996 auf diesen Unterschied hin. ${ }^{14}$ Obwohl die Black-Rice-Hypothese auch kritisch hinterfragt wurde, wird die Grundannahme, dass versklavte Menschen aus Afrika mit mehr als rein physischer Arbeitskraft aufwarten konnten, mittlerweile weitgehend akzeptiert. ${ }^{15}$ Relevant für diese Aspekte ist auch die Historikerin Edda Fields-Black, die sich auf transnationale Geschichte von Reisanbau in Westafrika sowie South Carolina und Georgia spezialisiert. ${ }^{16}$ Von Bedeutung sind zudem Archäologe Andrew Agha und Historiker Peter A. Coclanis, die sich ebenfalls mit der Geschichte der Reiskultivierung bzw. -industrie in den USA befassen. ${ }^{17}$

Der folgende Beitrag setzt sich im Anschluss an diese Forschungsdebatte kritisch mit dem Technologie- und Wissenstransfer des Reisanbaus von Westafrika nach Nordamerika, insbesondere nach South Carolina, auseinander. Es wird der Frage nachgegangen, warum und inwiefern in South Carolina Reisanbau- und Bewässerungssysteme nach westafrikanischem Vorbild angelegt wurden, und wie sich diese auf Produktion sowie Handel und Umsatz auswirkten. Darüber hinaus wird diskutiert, wie sich die Abschaffung der Sklaverei im Jahr 1865 auf die Reisindustrie in South Carolina auswirkte und ob es in der Folge zu einer Weiter- oder gar Rückentwicklung der Anbautechniken kam. Schließlich wird untersucht, wann asiatischer Reis als Konkurrenzprodukt in Europa auf den Markt kam, wo die Nachfrage an Reis besonders hoch war. ${ }^{18}$ Berücksichtigt wird auch Georgia, wo das Getreide ebenfalls in großen Mengen angebaut wurde. So

11 Edda L. Fields-Black, Atlantic rice and rice farmers. Rising from debate, engaging new sources, methods, and modes of inquiry, and asking new questions, in: Atlantic Studies 12 (2015), Nr. 3, S. 276-295, hier S. 277, [https:// www.tandfonline.com/doi/full/10.1080/14788810.2015.1068080], eingesehen 15. 8. 2018.

12 David Eltis/Philip Morgan/David Richardson, Agency and Diaspora in Atlantic History. Reassessing the African Contribution to Rice Cultivationin the Americas, in: The American Historical Review 112 (2007), Nr. 5, S. 1329-1358, hier S. 1332, [https://www.jstor.org/stable/40007098], eingesehen 27. 7. 2018.

13 Walter Hawthorne, The Cultural Meaning of Work. The "Black Rice Debate" Reconsidered, in: Dagmar Schaefer/ Edda L. Fields-Black u. a. (Hrsg.), Rice. Global networks and new histories, Cambridge 2015, S. 279-290, hier S. 280.

14 Judith Carney, Landscapes of Technology Transfer. Rice Cultivation and African Continuities, in: Technology and Culture 37 (1996), Nr. 1, S. 5-35, hier S. 5, [https://www.jstor.org/stable/3107200], eingesehen 27. 7. 2018.

15 Kenneth G. Kelly, Rice and its consequences in the greater "Atlantic" world, in: Atlantic Studies 12 (2015), Nr. 3, S. 273-275, hier S. 273, [https://doi.org/10.1080/14788810.2015.1062699], eingesehen 7. 2. 2019.

16 Carnegie Mellon University, Edda L. Fields-Black, 2019, [https://www.cmu.edu/dietrich/history/people/faculty/ fields-black.html], eingesehen 7. 2. 2019.

17 UNC College of Arts \& Sciences, Peter A. Coclanis, o. D., [http://history.unc.edu/people/faculty/peter-coclanis/], eingesehen 27. 7. 2018.

18 Carney, Out of Africa, S. 141. 
wurden im Jahr 1860 in den USA 187,2 Millionen Pfund davon produziert, davon 63,6 Prozent in South Carolina, 28 Prozent in Georgia. ${ }^{19}$ Das entspricht einer Gesamtproduktion von knapp 85.000 Tonnen Reis.

In der deutschsprachigen geschichtswissenschaftlichen Forschung kam der Thematik bisher kaum Aufmerksamkeit zu. Bei der vorliegenden Arbeit handelt es sich um einen Versuch, eurozentrische geschichtswissenschaftliche Standpunkte zu überwinden und sich stattdessen einer globalgeschichtlichen Perspektive anzunähern.

\section{Technologie- und Wissenstransfer des Reisanbaus von Westafrika nach South Carolina}

Ziel des folgenden Kapitels ist es, aufzuzeigen, wann und wie es erstmals zum Anbau von Reis im Südosten Nordamerikas kam, und wie sich die Produktion und der globale Export in der Folgezeit entwickelten. Dies soll anhand einer beispielhaften Auswahl an Quellen aufgezeigt werden. ${ }^{20}$

In keiner anderen Region in den USA spielte Reis eine für die Wirtschaft so wichtige Rolle wie in South Carolina, wo er, neben anderen Lebensmitteln, ab den 1670er-Jahren u. a. von Sklav*innen für ihren Eigenbedarf angebaut wurde. Bereits zwanzig Jahre später wurde das Getreide in großen Mengen als Exportware produziert. ${ }^{21}$ In seinem Artikel "Of the Introduction of Rice and Tar in Our Colonies" aus dem Jahr 1766 schreibt P. Collinson, dass seit Ende des 17. Jahrhunderts Reisimporte stark gefördert wurden, der Anbau aber zu Beginn einige Hürden zu überwinden hatte. Die Menschen waren mit den Methoden des Anbaus nicht vertraut, Fortschritte machten sich in den ersten neun bis zehn Jahren nur langsam bemerkbar und die produzierten Mengen reichten für den Inlandsverbrauch jener Zeit noch nicht aus. Collinson streicht vor allem die Plantagenbesitzer als jene Akteure heraus, die als erste in South Carolina mit Reis experimentiert hätten. Allerdings kann vermutlich nicht davon ausgegangen werden, dass er auch versklavte Menschen befragte, wann genau sie begannen, die Pflanze anzubauen.22 Der Anbau in South Carolina entwickelte sich ab 1690 rasant und bereits gegen Ende des 18. Jahrhunderts galt der spätere US-Bundesstaat als eine der weltweit reichsten landwirtschaftlichen Regionen ${ }^{23}$ und als wohlhabendste Plantagenregion Nordamerikas. ${ }^{24}$ Collinson beschreibt:

"[l]t soon became a staple commodity; it is therefore very probable, that in the years 1715 or 1716, a quantity was raised sufficient for exportation, which continued to increase till the 1726, and then it became a great article of commerce."25

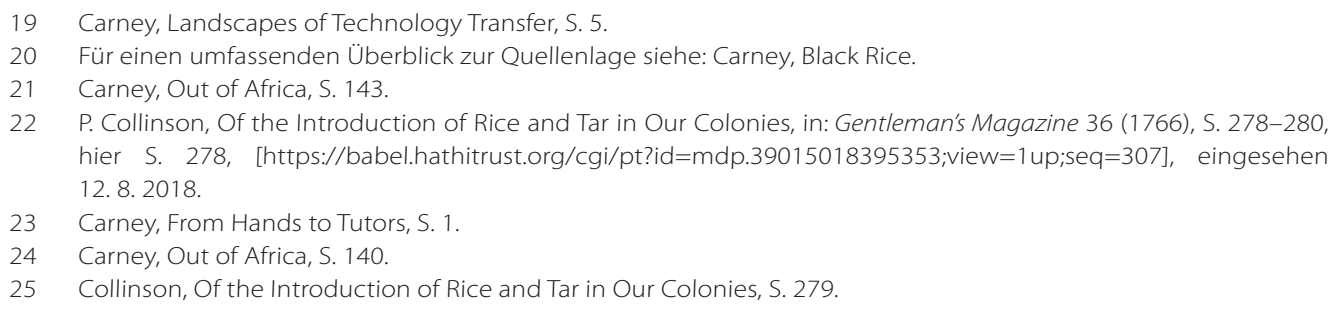

25 Collinson, Of the Introduction of Rice and Tar in Our Colonies, S. 279 
Er erwähnt außerdem ein Schreiben von Sam Eveligh, einem Händler aus Charles Town, dem heutigen Charleston, in dem sich eine kontinuierliche Steigerung der Exportmenge erkennen lässt. Während die Anzahl der exportierten Reisfässer in den Jahren 1726/1727 bei 40.000 liegt, erhöht sich die Exportmenge bis 1760/1761 um das 2,5-fache auf 100.024 Stück. Ob es sich um die Exportmenge South Carolinas, Charles Towns oder Sam Evelighs handelt, geht nicht eindeutig aus der Quelle hervor. Zweifelsfrei belegt ist jedoch die Steigerung der Exporte.

In den Jahren 1761/1762 ist in der Tabelle ein starker Rückgang der Ausfuhrmengen verzeichnet, den Collinson auf eine Hinwendung zur Produktion des Farbstoffes Indigo zurückführt. ${ }^{26}$ Neben Reis und Indigo wurde auch Baumwolle produziert, wobei die beiden ersten eine besonders wichtige Rolle für South Carolinas Wirtschaft spielten. ${ }^{27}$ Indigo wurde in South Carolina ab den späten 1740er-Jahren produziert und entwickelte sich bis in die 1770er-Jahre zum zweitwichtigsten Exportgut nach Reis. Laut R. C. Nash waren es Reis und Indigo, die das rasche Wirtschaftswachstum South Carolinas hervorriefen und die Entwicklung zur reichsten Plantagenregion Nordamerikas ermöglichten. ${ }^{28}$ Allerdings stiegen nicht nur die Produktions- und Exportmengen sukzessive an, sondern auch die Zahl der importierten Sklav*innen. Im Jahr 1680 wurden 200 versklavte Afrikaner*innen nach South Carolina gebracht: Diese Zahl stieg im Jahr 1770 auf mehr als 75.000 Menschen und erreichte ihren Höhepunkt 1860 mit mehr als 400.000 Personen. ${ }^{29}$ Vor allem versklavte Menschen aus den Gebieten zwischen dem Senegal und der Elfenbeinküste, wo westafrikanischer Reis (Oryza glaberrima) beheimatet ist, brachten laut Wood, Littlefield und Carney ihr Wissen und ihre landwirtschaftlichen Techniken nach Amerika: ${ }^{30}$

"The grain's arrival in the American Atlantic and role in plantation societies represent a remarkable form of technology transfer from West Africa under conditions of forced labor that are difficult to imagine." ${ }^{\prime 31}$

Wood ist der Ansicht, dass Reis höchstwahrscheinlich erstmals von Sklav*innen zur Eigenverpflegung angebaut wurde, da jene mit der Pflanze und deren Anbau und Bewirtschaftung vertraut waren - im Unterschied zu englischen und französischen Kolonialist*innen. ${ }^{32}$ Er war somit der erste Historiker, der afrikanische Zwangsarbeit nicht auf physische Arbeitskraft reduzierte, sondern landwirtschaftliches Wissen undTechnologien als Grundstein der Reisindustrie in South Carolina sah. ${ }^{33}$ Eine ähnliche Ansicht wird einige Jahre später von Littlefield vertreten:

26 Collinson, Of the Introduction of Rice and Tar in Our Colonies, S. 279.

27 Edward Mercer, Colonial Carolinass Natural Resources, 2018, 1.9.2018, [https://sciencing.com/colonial-carolinasnatural-resources-8329601.html], eingesehen 12. 11. 2018.

28 R. C. Nash, South Carolina and the Atlantic Economy in the Late Seventeenth and Eighteenth Centuries, in: The Economic History Review 45 (1992), Nr. 4, S. 677-702, hier S. 679-680, [https://www.jstor.org/stable/2597414], eingesehen 7. 2. 2019.

29 Bernd Stöver, United States of America. Geschichte und Kultur. Von der ersten Kolonie bis zur Gegenwart, München 20132', S. 205.

30 Carney, From Hands to Tutors, S. 1-2.

31 Carney, Out of Africa, S. 147

32 Carney, Black Rice, S. 80.

33 Carney, From Hands to Tutors, S. 1-2. 
"Africans were able to give technical advice and skill, which Europeans not only accepted but actually sought. Even more than is commonly assumed, the economic and social structures of American slave societies were, therefore, a mutual accomplishment.".34

Carney schließt in ihrem Essay "Out of Africa“ nicht nur auf den Technologietransfer von Westafrika nach Amerika, sondern stellt auch die Frage, ob westafrikanische Sklav*innen gezielt von den Plantagenbesitzer angeheuert wurden, um beim Reisanbau, mit dem sie vertraut waren, zu helfen, oder ob er von den versklavten Menschen erstmals eingeführt wurde und ursprünglich rein für deren Eigenbedarf bestimmt war. ${ }^{35}$ Mehr als vierzig Prozent der nach South Carolina verschleppten Personen stammten aus Westafrika, ${ }^{36}$ wo afrikanischer Oryza glaberrima mit hoher Evidenz unabhängig von der in Asien domestizierten Reispflanze Oryza sativa seit mehr als 3000 Jahren $^{37}$ angebaut worden war. ${ }^{38}$ Diese Annahme wird von zahlreichen Quellen gestützt. ${ }^{39}$ Eine Chronik von Gomes Eanes de Azurara enthält u. a. einen Bericht des portugiesischen Seemanns Stevam Alfonso aus dem Jahr 1444, wo beschrieben wird:

„They arrived sixty leagues beyond Cape Verde, where they met with a river which was of good width, and into it they entered with their caravels ... they found much of the land sown, and many fields sown with rice... And he said that land ... seemed like marsh." ${ }^{40}$

Fields-Black fasst in einem neueren Essay zusammen, dass die Forschung mittlerweile weitgehend in der Annahme der Eigenanbau-These übereinstimmt. ${ }^{41}$ Aufgrund fehlender Quellen ist es jedoch nicht möglich zu beurteilen, ob es sich bei der Selbstversorgungskultivierung um den Vorläufer der Reisindustrie in South Carolina handelte oder ob die Plantagenbesitzer*innen die Reisproduktion initiierten. ${ }^{42}$ Das heißt, die Frage, welche Rolle westafrikanische Sklav*innen an der Entstehung und Entwicklung der Reiskultivierung in der Plantagenwirtschaft spielten, bleibt weiterhin offen. ${ }^{43}$

Gegen Ende des 17. Jahrhunderts wurden Techniken des Reisanbaus und dessen Verarbeitung von versklavten Menschen, die laut Fields-Black höchstwahrscheinlich aus Westafrika stammten, übernommen. ${ }^{44}$ Ein Beispiel für diese Techniken ist das Verwenden von trunks, dabei handelt es sich um Schleusen, mit denen die Bewässerung der

\footnotetext{
34 Littlefield, Rice and Slaves, S. 177.

35 Carney, Out of Africa, S. 141-142.

36 Carney, From Hands to Tutors, S. 4.

37 Muhua Wang/Yeisoo Yu u. a., The genome sequence of African rice (Oryza glaberrima) and evidence for independent domestication, in: Nature Genetics 46 (2014), Nr. 9, S. 982-991, hier S. 982, [https://www.nature.com/ articles/ng.3044.pdf], eingesehen 7. 2. 2019.

38 Ebd., S. 987

39 Eine hervorragende Auswahl befindet sich in Carney, Black Rice.

40 Gomes Eanes de Azurara, The Chronicle of the Discovery and Conquest of Guinea, London 1899, S. 263-264, zit. n. Carney, Black Rice, S. 13.

41 Fields-Black, Atlantic rice and rice farmers, S. 278.

42 Ebd., S. 286

43 Ebd., S. 289

44 Ebd., S. 278
} 
Felder reguliert werden konnte. ${ }^{45}$ Weitere übernommene Techniken waren das Pflanzen der Reissamen mithilfe von Fersen und Zehen sowie für die Ernte und Verarbeitung hilfreiche Gegenstände wie Mörser, Stößel und spezielle Körbe, ${ }^{46}$ mit denen das Getreide geschält wurde. ${ }^{47}$ Ab dem 18. Jahrhundert steigerten die Plantageneigentümer die Produktivität, indem sie Bewässerungs- und Weiterverarbeitungstechnologien zunehmend mechanisierten, um die Ernteerträge zu erhöhen und die Nachfrage zu befriedigen. Das Säen von Reissamen mithilfe von Fersen und Zehen hingegen wurde bis ins 20. Jahrhundert praktiziert. ${ }^{48}$

\section{Reisanbausysteme in der Praxis}

Das nachfolgende Kapitel verfolgt das Ziel, die verschiedenen Reisanbausysteme, die sowohl in Westafrika als auch in South Carolina praktiziert wurden, zu beschreiben und in ihren historischen Kontext einzubetten, wobei zunächst kurz auf die geografischen Gegebenheiten South Carolinas eingegangen werden soll.

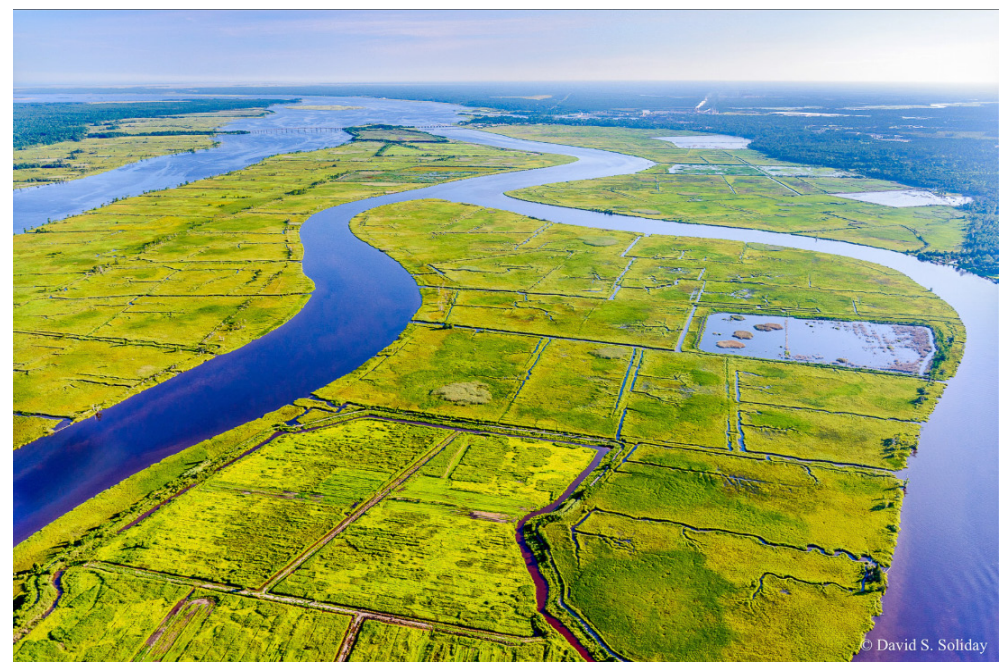

Abbildung 1: Ehemalige Reisfelder in Georgetown/Black River, Great Pee Dee \& Waccamaw River Mit freundlicher Genehmigung von (c) David S. Soliday.

South Carolinas Landschaften sind vom Wasser geprägt und bestehen aus moosbewachsenen Mooren, Süßwasserflüssen und Bächen, Barriereinseln und flachem, feuchtem Sumpfland. Zwischen den Inseln, die parallel zur Küste liegen, und den Sümpfen befinden sich tiefere Mündungsgebiete. Aktuelle Luftaufnahmen, wie beispielsweise jene vom Fotografen David S. Soliday, ${ }^{49}$ lassen erkennen, wie die Menschen die Umwelt

45 Hayden R. Smith, Reserving Water. Environmental and Technological Relationships with Colonial South Carolina Inland Rice Plantations, in: Schaefer/Fields-Black u. a. (Hrsg.), Rice., S. 189-211, hier S. 199.

46 Fields-Black, Atlantic rice and rice farmers, S. 278.

47 The National Museum of American History, Rice Fanner Basket, Mt. Pleasant, South Carolina, 1970s, O. D., [http:// americanhistory.si.edu/collections/search/object/nmah_659983], eingesehen 12. 11. 2018.

48 Fields-Black, Atlantic rice and rice farmers, S. 279.

49 An dieser Stelle möchte ich David S. Soliday für die Erlaubnis, eine seiner Luftaufnahmen zu verwenden, herzlich danken. 
nach ihren Bedürfnissen umgeformt haben. Entlang der Flüsse stechen, wie in Abbildung 1 ersichtlich, zahlreiche rechteckige Flächen ins Auge, bei denen es sich um ehemalige Reisfelder handelt, die ursprünglich durch Zwangsarbeit angelegt wurden und durch Baumstämme, Deiche und Kanäle begrenzt sind. Die Umwelt wurde dadurch verändert und den landwirtschaftlichen, ökologischen und ökonomischen Interessen der Menschen angepasst. ${ }^{50}$

Mit der Konstruktion von künstlichen Böschungen beschäftigt sich der Archäologe Andrew Agha. Er untersuchte Böden in und um die von Sklav*innen gefertigten Aufschüttungen und stieß dabei nicht nur auf materielle Quellen, wie zum Beispiel Nägel, diverse Überreste von Tabakpfeifen, Keramik- und Porzellanwaren, ${ }^{51}$ sondern konnte auch von der heutigen Beschaffenheit der Böschungen auf frühere Arbeitstechniken schließen. So wurde z. B. Ton verwendet, um die Wälle zu stabilisieren.52 In Westafrika wurden bereits seit Jahrhunderten Tonwaren angefertigt. Daher warf Agha die Frage auf, ob afrikanische Töpfer*innen am Bau von künstlichen Böschungen und Wällen beteiligt gewesen sein könnten. ${ }^{53}$ Wenn das Wissen und die Technologien von dort stammen, könnte dies auch für den Reisanbau gelten: ${ }^{54}$ "If historians have shown rice connections between South Carolina and the Guinea coast, then further studies of early inland rice fields could reveal evidence of technological transfer." ${ }^{\prime 55}$

Drei Reisanbausysteme fanden sowohl in Westafrika als auch in South Carolina Anwendung. Dabei handelte es sich um das Hochlandsystem, den Inland-Nassreisanbau und den gezeitenabhängigen Küstenreisanbau. ${ }^{56}$ Nachfolgend werden insbesondere die zum Anlegen der Felder nötigen Eingriffe in die Natur beschrieben sowie die jeweilige Arbeitsintensität und Produktivität geschildert. Im Anschluss wird eine Quellenanalyse durchgeführt.

\subsection{Hochlandsystem}

Reis wurde von europäischen Kolonialisten zunächst in South Carolinas Hochland angebaut. Diese Methode orientiert sich am Anbau von Weizen: Das Getreide wurde auf trockenem Boden angepflanzt, für die Bewässerung sorgten Niederschläge. Bäume wurden zunächst abgeholzt, anschließend Felder angelegt und die Reissamen eingepflanzt. ${ }^{57}$ Eine anschauliche Beschreibung lieferte John Norris im Jahr 1712 in seinem Pamphlet „Profitable Advice for Rich and Poor...", in dem die fiktive Figur Simon

50 Jodi A. Barnes/Rebekah Dobrasko, South Carolina's Tidal Rice Fields. Consultation, Collaboration, and Cultural Landscapes, in: International Journal of Cultural Property 21 (2014), Nr. 4, S. 423-444, hier S. 425-426.

51 Andrew Agha, Standing the test of time. Embankment investigations, their implications for African technology transfer and effect on African American archaeology in South Carolina, in: Atlantic Studies 12 (2015), Nr. 3, S. 336-354, hier S. 339, [https://www.tandfonline.com/doi/full/10.1080/14788810.2015.1062324], eingesehen 15. 8. 2018.

52 Ebd., S. 346.

53 Ebd., S. 349

54 Ebd., S. 350.

55 Ebd.

56 Carney, Black Rice, S. 84-85.

57 John Norris, Profitable Advice for Rich and Poor, 1712, zit. n. Barnes/Dobrasko, South Carolina's Tidal Rice Fields, S. 428 . 
Question den Plantagenbesitzer James Freeman über die Besiedlung South Carolinas befragt: ${ }^{58}$

"As to the manner of planting our rice, after the land is clear'd ... we, with hoes, trench the land something like furrows made with a plough but not so deep and about a foot distance between each trench; and when the land is so trench'd, in the month of April we feed it, carefully, within each trench, and cover it thin with earth, one peck and half is sufficient for to feed an acre, then, with narrow hoes made for the purpose ... we hoe, weed, or cut up the grass, or other trash, growing between the said trenches of rice, which ought carefully to be done three times in the summer, for grass and weeds growing between the corn, pease, or rice, will otherwise destroy or spoil the crop: then, at harvest, which comes in September, we reap and carry it to barns, which when trash'd, if it prov'd a good crop, 30, 3, or 40 bushels, sometimes more.".59

Die Produktivität des Hochlandsystems hing besonders von den klimatischen Bedingungen ab. Je höher die Niederschlagsmengen, desto günstiger war das für den Reisanbau. ${ }^{60}$ Auch wenn das Roden des ausgewählten Geländes zeitaufwändig war, so war das Bewirtschaften der Felder dennoch weniger arbeitsintensiv als beim Nassreisanbau. ${ }^{61}$ Trotz dieser Vorteile erwies sich die Anbaumethode als vergleichsweise wenig produktiv und wurde ab 1720 sukzessive vom Inland-Nassreisanbau abgelöst. ${ }^{62}$

\subsection{Inland-Nassreisanbau}

Zentral für den Erfolg des Inland-Nassreisanbaus waren Lage und Wasserfluss. ${ }^{63}$ Sklav*innen legten Reisfelder und Wasserspeicher an, um je nach Bedarf bewässern zu können. Die natürliche Umgebung musste dafür gegebenenfalls angepasst werden, ${ }^{64}$ etwa durch das Errichten von Kanälen. Wichtige Charakteristika waren slow water permeability und high water-holding capacity, was bedeutet, dass das Wasser in den Feldern und Speichern blieb, anstatt im Boden zu versickern. ${ }^{65}$ Dieses Reisanbausystem wurde für Westafrika u. a. von René Caillié im frühen 19. Jahrhundert beschrieben:

"As the country is flat, they take care to form channels to drain off the water. When the inundation is very great, they take advantage of it and fill their little reservoirs, that they may provide against the drought and supply the rice with the moisture which it requires." ${ }^{16}$

58 Alexander Moore, Rezension zu: Selling a New World. Two Colonial South Carolina Promotional Pamphlets by Thomas Nairne and John Norris by Thomas Nairne, John Norris and Jack P. Greene, in: The South Carolina Historical Magazine 90 (1989), Nr. 4, S. 324-426, hier S. 325, [https://www.jstor.org/stable/27568096], eingesehen 7. 2. 2019. John Norris, Profitable Advice for Rich and Poor, 1712, zit. n. Barnes/Dobrasko, South Carolina's Tidal Rice Fields, S. 427.

60 Apichart Vanacichit, Facts about Rice, in: Sontag (Hrsg.), Rice processing, S. 11-24, hier S. 13.

61 Carney, Black Rice, S. 57-58.

62 Smith, Reserving Water, S. 191.

63 Ebd., S. 189-193.

64 Ebd., S. 193.

65 Ebd., S. 196

66 Carney, Black Rice, S. 22. 
Auch wenn dieses Zitat den Inland-Nassreisanbau nicht vollständig beschreibt, so ist doch die Trennung zwischen den Feldern und Wasserspeichern ein wichtiges Merkmal dieser Art der Reisproduktion. Um den bestmöglichen Ertrag zu erzielen, mussten die für den Inland-Nassreisanbau günstigsten Böden und Umgebungen ermittelt werden. Als geeignet beschrieb James Glenn 1761:

"[T] he best land for rice is a wet, deep, miry soil; such as is generally to be found in Cypress Swamps; or a black greasy Mould with a Clay foundation; but the very best lands may be meliorated by laying them under water at proper Seasons." ${ }^{16}$

Inland-Nassreisanbau war aufgrund der Wasserspeicher nicht mehr von der Niederschlagshöhe abhängig. Um die Bewässerung regulieren zu können, wurden trunks (Schleusen) verwendet.68

\subsection{Gezeitenabhängiger Küstenreisanbau}

Beim gezeitenabhängigen Küstenreisanbau wurden Ebbe und Flut von Flüssen zur Bewässerung der Reisfelder genutzt. ${ }^{69}$ Zunächst legten die Sklav*innen Deiche und sekundäre Kanäle für Flusswasser um rechteckige Flächen an, die anschließend eingedämmt wurden, um ein Überlaufen zu verhindern. Die Dämme verfügten über Schleusen, mit denen der Wasserstand der Feldabschnitte reguliert werden konnte. Wenn diese während der Flut geöffnet wurden, wurde das Feld überschwemmt - blieben sie geschlossen, verblieb das Wasser auf dem Feld. Sobald sie bei Ebbe geöffnet wurden, konnte das Wasser abfließen. Es handelte sich um ein überaus ausgeklügeltes System der Wasserkontrolle. ${ }^{70}$ Da das Anlegen der Felder arbeits- und zeitintensiv war, wurde eine hohe Anzahl von Arbeitskräften benötigt. Dies würde die rasant steigende Einfuhr von Sklav*innen nach South Carolina erklären, wobei berücksichtigt werden muss, dass auch die Anzahl der Plantagen und die Nachfrage nach Reis stiegen. ${ }^{71}$ Besonders durchdringend wurde Küstenreisanbau ab etwa 1750 betrieben. Die größten Flüsse South Carolinas, die ins Meer münden, wurden nach und nach von Reisfeldern gesäumt. Die Anbauflächen befanden sich idealerweise nicht zu nahe am Meer und nicht zu weit flussaufwärts. So konnte Salzwasser als Staudamm für die Strömung der Süßwasserflüsse genutzt werden. ${ }^{72}$

67 James Glen, 1761, zit. n. Smith, Reserving Water, S. 196

68 Carney, Black Rice, S. 58.

69 Barnes/Dobrasko, South Carolina's Tidal Rice Fields, S. 428

70 Carney, Black Rice, S. 92.

71 Smith, Reserving Water, S. 192.

72 Edda L. Fields-Black, Deep Roots. Rice farmers in West Africa and the African diaspora, Bloomington 2008, S. 158. 


\section{Exemplarische Quelle: South Carolina. Reportage im „Harper's New Monthly Magazine“ über „The Rice Lands of the South”}

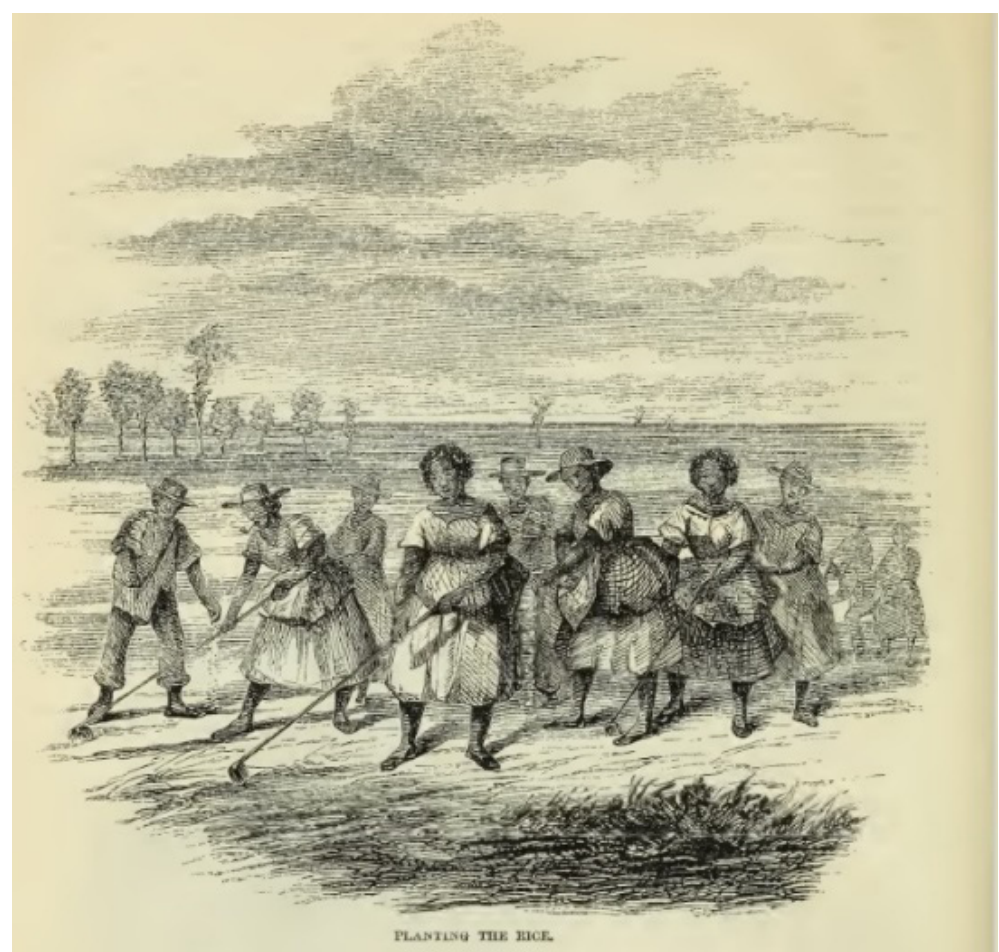

Abbildung 2: Pflanzen von Reis, The Rice Lands of the South, Harper's New Monthly Magazine 19, S. 726.

In diesem Abschnitt soll eine Quelle, bestehend aus einer Abbildung und einer im Jahr 1859 im „Harper's New Monthly Magazine“ erschienenen und von T. Addison Richards verfassten Reportage analysiert werden. Im Zentrum der Abbildung befinden sich afrikanisch-stämmige Arbeiter*innen, die beim Bearbeiten des Bodens mit Pflügen und Hacken dargestellt werden. Beschrieben wird in der zugehörigen Reportage das Anpflanzen von Reis. Richards schildert zunächst den gezeitenabhängigen Küstenreisanbau: Nach dem Anlegen der Felder inklusive Rodungen sowie dem Errichten von Wällen, Deichen, Böschungen, Ventilen und Wasserschleusen wurde der Boden mit Pflügen und Hacken für das Säen der Samen in ausgehobene Rinnen und Furchen vorbereitet. Eine weitere Arbeitskraft bedeckte die Samen wiederum leicht mit Erde. Das Säen/Anpflanzen musste laut Richards an einem einzigen Tag abgeschlossen werden, damit bei der nächsten Flut die Schleusen geöffnet und die Felder gleichmäßig bewässert werden konnten. ${ }^{73}$ Es wird deutlich, wie arbeits- und zeitaufwändig gezeitenabhängiger Küstenreisanbau war. Er beschreibt in seiner Reportage aber nicht nur den Anbau des Getreides, sondern auch die Reispflanze, Ernte und Verarbeitung, die geografischen Faktoren von South Carolina und Georgia, sowie die Gesundheit der 
Zwangsarbeiter*innen, wobei Richards Ausführungen verdeutlichen, wie abwertend Menschen mit dunkler Hautfarbe angesehen wurden, die sprachlich eher die Ebene eines Nutztieres als eines Menschen einnehmen:

"To cultivate these lands by white labor, if practicable at all, would be, unquestionably, at an immense sacrifice of life. Even the African, who seems to be physically so well adapted to the climate, does not altogether escape. [...] Such immunity as he does enjoy from the subtle poison of the malaria, which fills all the air around him, may be ascribed to his exemption from all but comparatively light labor, and the good care, both preventive and curative, which is ever taken of him. The master never resides on the fields in summer time; he would as soon think, and very reasonably, of facing a rifle-shot.".74

Aus diesem Abschnitt geht Folgendes hervor: Während die Sklav*innen täglich auf den Feldern Zwangsarbeit verrichteten, besuchten die Plantagenbesitzer*innen während der heißen Sommermonate die Felder nicht. Argumentiert wird mit der körperlich besseren Eignung sowie der erhöhten Immunität gegen Malaria der afrikanisch-stämmigen Arbeiter*innen, die in den Reisfeldern vergleichsweise leichte Arbeiten verrichten würden und auf deren Gesundheit gut Acht gegeben würde. Dies widerspricht jedoch einer Aussage Fields-Blacks, die auf die Machtausübung und Anwendung von Gewalt in den Feldern hinweist, um die versklavten Menschen zur Arbeit zu zwingen, ${ }^{75}$ und ist vermutlich als Euphemismus zu werten. Mit der Sorge der Plantagenbesitzer, bald einem Gewehrlauf ins Auge zu blicken, ist hinsichtlich des Kontexts vermutlich nicht von Revolten der Sklav*innen die Rede, sondern vom immens hohen Risiko, im Sommer an Malaria zu erkranken. Gegen Mitte des 18. Jahrhunderts, als die Wohnhäuser noch neben den Reisfeldern errichtet wurden, starben daran zahlreiche weiße Siedler*innen (und versklavte Menschen). Gegen Ende des 18. Jahrhunderts verzogen die Eigentumsparteien daher in die Städte und mieden die Felder im Sommer. Die versklavten Zwangsarbeiter*innen waren jedoch keinesfalls immun: Deren Mortalität und insbesondere auch die Kindersterblichkeit waren exorbitant hoch.7 ${ }^{76}$

\section{Niedergang der Reiswirtschaft in South Carolina und heutige Flächennutzung}

Im folgenden Kapitel wird der Frage nachgegangen, wie sich die Abschaffung der Sklaverei nach dem Ende des amerikanischen Bürgerkrieges auf die Reisindustrie in South Carolina auswirkte. Es soll insbesondere herausgearbeitet werden, ob es zu Weiter- und/oder Rückentwicklung der Reisanbautechniken kam, und ob und wann das Aufkommen von in Asien produziertem Reis als Konkurrenz am Markt eine Rolle für die Reisindustrie South Carolinas spielte.

\footnotetext{
74 Richards, Rice Lands, S. 731.

75 Fields-Black, Atlantic rice and rice farmers, S. 288.

76 Ebd., S. 287-288.
} 
Kurz bevor es zum amerikanischen Bürgerkrieg (1861-1865) kam, lebten in den Südstaaten knapp vier Millionen versklavte Menschen. ${ }^{77}$ Ein für den Bürgerkrieg ausschlaggebendes Ereignis war die Wahl Abraham Lincolns, der die Abschaffung der Sklaverei forderte, zum US-amerikanischen Präsidenten. ${ }^{78}$ Durch den Sezessionskrieg wurden weite Gebiete Nordamerikas verwüstet und der wirtschaftliche Schaden für den Süden war beträchtlich - nicht zuletzt, weil die zahlreichen versklavten Menschen freigelassen werden mussten. ${ }^{79}$ Im 19. Jahrhundert kam es zu einer grundlegenden Veränderung und Verschiebung der US-Reisindustrie, die zuvor von South Carolina und Georgia dominiert worden war und die vor allem die Nachfrage auf europäischen Märkten befriedigte.

Coclanis kritisiert, dass der Untergang der Reisindustrie in diesen Gebieten von vielen Historiker*innen vor allem mit dem Ausbruch des Bürgerkriegs 1861 in Verbindung gebracht wird. Er nennt drei Aspekte, die für ihn als Erklärung des Untergangs der Reisindustrie zu kurz greifen: die Zerstörung der Reisfelder durch den Krieg, Schwierigkeiten, die Reisindustrie ohne Sklaverei wiederzubeleben, und die Höhe der vom Sezessionskrieg verursachten Handelseinbußen. ${ }^{80}$ Coclanis erläutert, dass die globalen Veränderungen des Reisangebots, wie beispielsweise die Marktöffnung für verhältnismäßig billigen asiatischen Reis auf dem europäischen Markt ab den 1790er-Jahren, außer Acht gelassen wurden. ${ }^{81}$ Aufgrund der Konkurrenz aus Asien gingen die amerikanischen Marktanteile ab 1800 zurück und schon am Vorabend des Bürgerkriegs um 1860 waren die Marktanteile der Atlantiksüdstaaten vergleichsweise gering. Daraufhin wurde versucht, Reis vermehrt nach Kuba oder Puerto Rico zu exportieren, allerdings führte dies nicht zum gewünschten Erfolg. Ab spätestens 1879 konnten die USA mit der asiatischen Konkurrenz nicht mehr mithalten und begannen, selbst Reis aus Asien zu importieren. ${ }^{82}$

\section{Exemplarische Quelle: Georgia. Zeitungsartikel im „Harper's Magazine“ über Reiskultivierung}

Bei der zweiten exemplarischen Quelle handelt es sich um einen von Alfred J. Waud verfassten Artikel mit Illustration der Zeitung „Harper's Magazine“, dem Nachfolger des "Harper's New Monthly Magazine". Erschienen ist die Ausgabe am 5. Juni 1867, bereits nach dem amerikanischen Bürgerkrieg und der Befreiung der Sklav*innen. Da es sich bei Georgia um die zweite wichtige Anbauregion von nordamerikanischem Reis handelte, wird anhand dieser Quelle zunächst untersucht, wie auf der Wardell Plantage, die sich am Ogeechee River in der Nähe von Savanna befand, das Getreide angebaut wurde, bevor anschließend auf einen möglichen Werbeaspekt für künftige Arbeitnehmer*innen der US-Reisindustrie eingegangen wird.

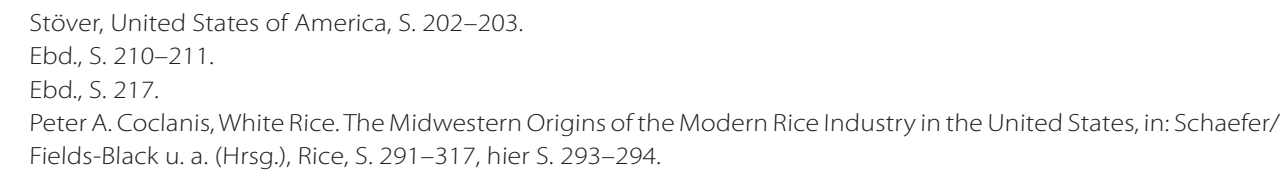




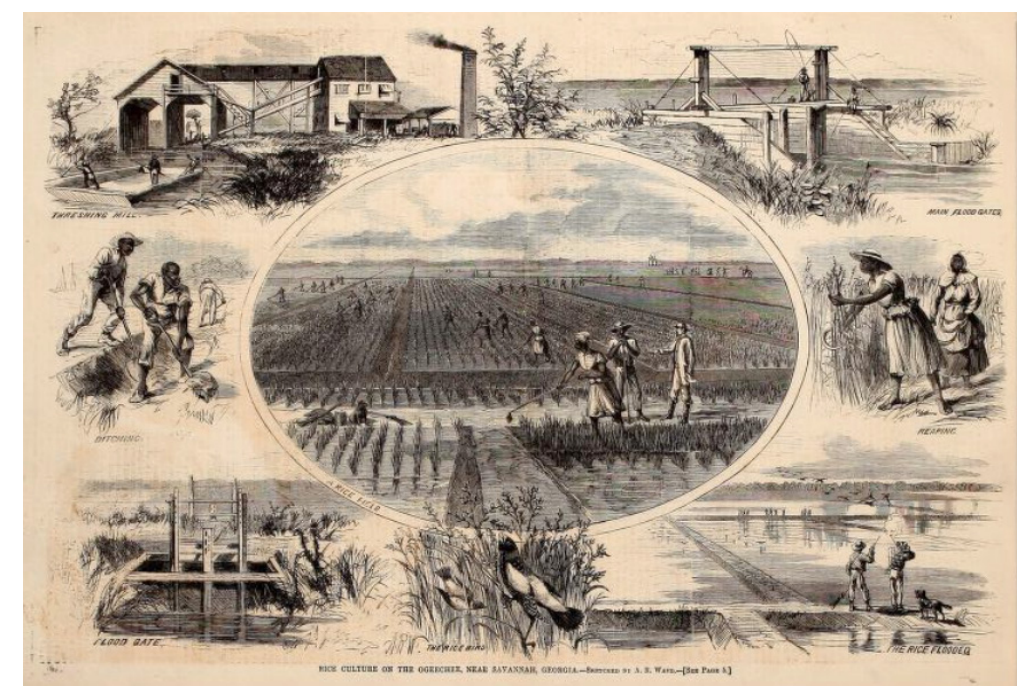

Abbildung 3: Sketches of a Rice Plantation, "Harper's Magazine," 5. Jänner 1867, S. 8.

Auch in dieser Quelle wird aufgrund der ausführlichen Beschreibung klar, dass es sich um gezeitenabhängigen Küstenreisanbau handelt. In der Mitte der Abbildung befinden sich Reisfelder, die bewirtschaftet werden. Links oben ist eine threshing-mill, eine Mühle, abgebildet, daneben befinden sich Wasserschleusen, die bei Flut das Bewässern der Reisfelder ermöglichen. Darunter ist zu erkennen, wie ein Bewässerungsgraben ausgehoben wird, rechts davon sind Frauen bei der Reisernte zu erkennen. Unten links wurden trunks gezeichnet, kleinere Tore aus ursprünglich ausgehöhlten Baumstämmen. Daneben befindet sich eine Zeichnung eines Reisstärlings, eines Vogels, der von besonderem Nutzen für die Produzent*innen war, da er die Felder von Insekten säuberte. ${ }^{83}$

Wie bei der zuvor erwähnten Reportage wird auch in diesem Artikel zunächst beschrieben, wie Reis angebaut wurde. In einem ersten Schritt werden die Felder ausgehoben, um eine Bewässerung zu ermöglichen. Anschließend werden sie händisch gepflügt und von Unkraut befreit. Nach dem Pflanzen werden sie für acht bis neun Tage geflutet. Sobald die Sprösslinge eine Höhe von zwei bis drei Zoll (das entspricht in etwa einer Höhe von fünf bis acht Zentimetern) erreichen, werden die Felder erneut bewässert. Das Wasser ernährt den Reis und verhindert gleichzeitig das Wachsen von Unkraut. Vor der Ernte wird das Wasser abgelassen. ${ }^{84}$ Auch in dieser Reportage wird erwähnt, wie viel leichter die Arbeit auf den Reisfeldern im Vergleich zu anderen Arbeiten sei:

„[...] their tasks are much lighter, usually being finished by the middle of the day, when they eat a good meal. To eat early makes them sick, and they often put it off till they have done their work [...] In their gardens they cultivate cabbage, potatoes, etc., and altogether lead a tolerably pleasant existence."85

83 Alfred Rudolph Waud, Sketches on a Rice Plantation, in: Harper's Magazine. A Journal of Civilization (1867), Nr. 523, S. 5-6, hier S. 6, [https://archive.org/stream/harpersweeklyv11 bonn\#page/4], eingesehen 22. 8. 2018.

84 Ebd., S. 5-6.

85 Ebd., S. 6. 
Der Auszug wirkt beinahe so, als ob der Autor Arbeitskräfte anwerben wolle, da der Artikel bereits zwei Jahre nach der Verabschiedung des Thirteenth Amendment gedruckt wurde. In diesem Sinn werden womöglich die vergleichsweise leichte Arbeit, die nur bis Mittag andauerte und viel Freizeit ermöglichte, und die "tolerably pleasant existence" genannt. ${ }^{86}$ Auf den Baumwollplantagen blieb die Arbeit der afrikanisch-stämmigen Bevölkerung auch nach dem Bürgerkrieg noch für eine Weile weitgehend unbezahlt. Dies könnte eventuell auf die Arbeit auf den Reisfeldern übertragen werden. ${ }^{87}$ In diesem Sinn würde der Autor die niedrig bezahlte Arbeit auf den Reisplantagen aufgrund der angenehmen Arbeitsverhältnisse rechtfertigen. Zudem muss bedacht werden, dass sich die amerikanische Reisindustrie zu jener Zeit bereits auf dem absteigenden Ast befand und im Vergleich zur asiatischen Reisindustrie ab spätestens 1879 nicht mehr konkurrenzfähig war. ${ }^{88}$

\section{Reisproduktion in den USA 1839-1919}

Die folgende Tabelle verdeutlicht die Verlagerung der US-amerikanischen Reisproduktion (in Millionen Pfund) im 19. und frühen 20. Jahrhundert: ${ }^{89}$

Tabelle 1: Reisproduktion in Millionen Pfund, adaptiert nach Coclanis, White Rice, S. 299.

\begin{tabular}{|l|l|l|l|l|l|l|l|l|l|}
\hline & $\mathbf{1 8 3 9}$ & $\mathbf{1 8 4 9}$ & $\mathbf{1 8 5 9}$ & $\mathbf{1 8 6 9}$ & $\mathbf{1 8 7 9}$ & $\mathbf{1 8 8 9}$ & $\mathbf{1 8 9 9}$ & $\mathbf{1 9 0 9}$ & $\mathbf{1 9 1 9}$ \\
\hline S. Carolina & 75,0 & 74,3 & 63,6 & 43,9 & 47,3 & 23,6 & 18,9 & 2,5 & 0,4 \\
\hline Georgia & 15,3 & 18,1 & 28,0 & 30,2 & 23,0 & 11,3 & 4,5 & 0,7 & 0,2 \\
\hline Louisiana & 4,5 & 2,0 & 3,4 & 21,5 & 21,1 & 58,8 & 69,0 & 49,6 & 45,3 \\
\hline Texas & 0,0 & 0,0 & 0,0 & 0,1 & 0,1 & 0,1 & 2,9 & 41,2 & 15,0 \\
\hline Arkansas & 0,0 & 0,0 & 0,0 & 0,1 & 0,0 & 0,0 & 0,0 & 5,9 & 19,2 \\
\hline Kalifornien & 0,0 & 0,0 & 0,0 & 0,0 & 0,0 & 0,0 & 0,0 & 0,0 & 19,6 \\
\hline
\end{tabular}

Während South Carolina und Georgia im Jahr 1839 noch 75 bzw. 15,3 Millionen Pfund Reis produzierten, sank die produzierte Reismenge stetig, wobei ein besonders starker Abfall zwischen 1879 und 1889 zu beobachten ist. Hierbei handelt es sich um den Zeitraum, in dem laut Coclanis die USA im Vergleich zur asiatischen Konkurrenz in Europa nicht mehr wettbewerbsfähig waren. In den Jahren 1899 bis 1919 kann die Verlagerung der US-Reisproduktion insbesondere nach Louisiana, Texas, Arkansas und Kalifornien deutlich beobachtet werden.

Nach Ende des Bürgerkrieges, der von 1862 bis 1865 andauerte, erwarben zahlreiche wohlhabende, in den Nordstaaten wohnende Menschen günstige Grundstücke in den Südstaaten und wandelten zahlreiche Plantagen in Jagdreservate um. ${ }^{90}$ Die Felder wurden ab dem frühen 20. Jahrhundert vorrangig mit dem Ziel bewirtschaftet, möglichst viele Wasservögel und heimische Wildtiere anzulocken, die sich als Jagdbeute für

86 Waud, Sketches, S. 6

87 Stöver, United States of America, S. 221.

88 Coclanis, White Rice, S. 299.

89 Ebd

90 Barnes/Dobrasko, South Carolina's Tidal Rice Fields, S. 424. 
Besitzer*innen und Besucher*innen der hunting lodges eigneten. Die ehemaligen Plantagengebäude wurden renoviert oder ausgebaut, neue Hütten und Lodges errichtet. Noch heute dienen viele ehemalige Reisfelder als Jagdgebiete. ${ }^{91}$ Zahlreiche einstige Anbaugebiete sind gegenwärtig im Besitz oder stehen unter der Verwaltung des U.S. Fish and Wildlife Service oder des South Carolina Department of Natural Resources, die diverse Bewilligungen für diese Areale erteilen.92 Die verschiedenen Projekte an dieser Stelle aufzulisten ist aus Platzgründen nicht möglich, auf den Websites der jeweiligen Institutionen finden sich jedoch genauere Informationen. ${ }^{93}$ Viele Flächen und Gebäude befinden sich weiterhin in privatem Besitz, und teilweise wird noch Reis angebaut. Um die ehemaligen Felder als historische und kulturelle Stätten zu wahren sowie die Bewirtschaftung und Nutzung zu regulieren wurden verschiedene Gesetze erlassen. ${ }^{94}$ Es kam nach dem Bürgerkrieg zu keinen technologischen Weiterentwicklungen mehr und insbesondere die Techniken zur Bewässerungsregulierung unterscheiden sich gegenwärtig wenig von jenen der Kolonialzeit. So werden beispielsweise noch immer trunks verwendet, lediglich einige Materialien wurden durch widerstandsfähigere ersetzt. ${ }^{95}$

\section{Fazit}

Die Forschungsfrage, warum und inwiefern in South Carolina Reisanbau- und Bewässerungssysteme nach afrikanischem Vorbild angelegt wurden, ist nicht eindeutig zu beantworten. Während P. Collinson im Jahr 1766 nur erwähnt, dass Plantagenbesitzer mit Reis experimentiert hätten,96 schließt Wood in den 1970er-Jahren erstmals, dass Reis höchstwahrscheinlich zunächst von der afrikanisch-stämmigen Bevölkerung angebaut wurde. ${ }^{97}$ Für diese These spricht auch, dass mehr als vierzig Prozent der nach South Carolina verschleppten Menschen aus Westafrika stammten, ${ }^{98}$ wo afrikanischer Oryza glaberrima seit mehr als 3.000 Jahren angebaut wurde. ${ }^{99}$ Auf diese Thesen bauten insbesondere Littlefield und Carney auf, wobei die Black-Rice-Hypothese von anderen Historiker*innen kritisch hinterfragt wurde. Allerdings fehlt eindeutiges Quellenmaterial, daher ist es derzeit nicht möglich zu beurteilen, welche Rolle westafrikanische Sklav*innen an der Entwicklung der Reisindustrie South Carolinas spielten. ${ }^{100}$ Die Forschung stimmt jedoch überein, dass Reis neben anderen Lebensmitteln angebaut wurde, und gegen Ende des 17. Jahrhunderts (west)afrikanische Techniken des Reisanbaus übernommen wurden. ${ }^{101}$ Beispiele für diese Techniken sind das Ver-

\footnotetext{
91 Barnes/Dobrasko, South Carolina's Tidal Rice Fields, S. 430.

92 Ebd., S. 440.

93 Informationen befinden sich auf den jeweiligen Websites: U.S. Fish and Wildlife Service, 21. 3. 2019 [https:// www.fws.gov/], eingesehen 7. 2. 2019; South Carolina Department of Natural Resources, 2019, [http://www.dnr. sc.gov/], eingesehen 7. 2. 2019.

94 Barnes/Dobrasko, South Carolina's Tidal Rice Fields, S. 438.

95 Ebd., S. 432.

96 Collinson, Of the Introduction of Rice and Tar in Our Colonies, S. 278.

97 Carney, Black Rice, S. 80

98 Carney, From Hands to Tutors, S. 4.

99 Wang/Yu u. a., The genome sequence of African rice (Oryza glaberrima) and evidence for independent domestication, S. 982.

100 Fields-Black, Atlantic rice and rice farmers, S. 289.

101 Ebd., S. 278.
} 
wenden von trunks, das Pflanzen der Reissamen mithilfe der Fersen und Zehen bis ins 20. Jahrhundert, oder die Nutzung von Mörsern, Stößeln und speziellen Körben. ${ }^{102}$ Ab dem 18. Jahrhundert begannen die Plantagenbesitzer, die Produktivität der Anbausysteme auszubauen und zu mechanisieren, um die Produktionszahlen zu steigern. ${ }^{103}$ Die Anstiege der Absatzzahlen, die South Carolina im 18. Jahrhundert mit den Worten Judith Carneys zur "wealthiest plantation economy in North America“104 aufsteigen ließen, lassen sich in Collinsons Artikel über die Reiswirtschaft im zweiten Kapitel dieser Abhandlung erahnen und waren mit dem Experimentieren und Mechanisieren der Anbausysteme in South Carolina und Georgia verbunden.

Der Untergang der Reisindustrie wird häufig mit den Folgen des amerikanischen Bürgerkriegs in Verbindung gebracht. Peter Coclanis schildert jedoch, dass auch andere Faktoren für den Einbruch der Reisindustrie im Südosten Nordamerikas verantwortlich waren. ${ }^{105}$ Auf den wichtigen europäischen Märkten kam bereits gegen Ende des 18. Jahrhunderts asiatischer Reis als Konkurrenzprodukt in Umlauf. ${ }^{106}$ Bereits vor Ausbruch des Bürgerkrieges hatten die Atlantiksüdstaaten große Marktanteile an Asien verloren und waren ab spätestens 1879 nicht mehr konkurrenzfähig. Die Reisindustrie verlagerte sich im Laufe der Zeit immer mehr nach Louisiana, Texas, Arkansas und Kalifornien, während in South Carolina heute nur mehr etwa 0,4 Prozent des US-amerikanischen Reises produziert werden. ${ }^{107}$ Um die Reisfelder als historische und kulturelle Stätten zu schützen, wurden diverse Gesetze erlassen, die dazu führten, dass es zu keinen technologischen Weiterentwicklungen mehr kam, sondern der Reisanbau und insbesondere dessen Bewässerungstechnologien in South Carolina heutzutage noch wie in der Kolonialzeit betrieben werden, ${ }^{108}$ wobei einige Materialien durch widerstandsfähigere ersetzt wurden. ${ }^{109}$

Obwohl sich seit den 1970er-Jahren zahlreiche Historiker*innen mit der Thematik beschäftigen, gibt es einige Forschungslücken. Aus einer E-Mail-Korrespondenz mit dem Fotografen David S. Soliday, ${ }^{110}$ dessen Arbeit insbesondere von Judith Carney wertgeschätzt und unterstützt wird, geht hervor, dass es sein nächstes Ziel sein wird, Reisfelder in Westafrika zu fotografieren und eine vergleichende Studie der Reiskultivierung im Südosten der USA und in Westafrika zu veröffentlichen. ${ }^{111}$ Dieses Projekt würde laut Carney die derzeit interdisziplinäre Forschung um eine Dimension ergänzen, die den

102 Fields-Black, Atlantic rice and rice farmers, S. 278

103 Ebd., S. 279

104 Carney, Out of Africa, S. 140.

105 Coclanis, White Rice, S. 293-296.

106 Ebd., S. 297.

107 Ebd., S. 299

108 Barnes/Dobrasko, South Carolina's Tidal Rice Fields, S. 438.

109 Ebd., S. 432.

110 David S. Solidays großartige Luftaufnahmen der ehemaligen Reisfelder sind einsehbar unter: David S. Soliday, o. D., [http://www.davidsoliday.com/rice/], eingesehen 23. 08. 2018.

111 Für die Umsetzung dieses umfangreichen, aber engagierten und für die Forschung überaus wichtigen und aufschlussreichen Projekts wünsche ich David S. Soliday viel Glück und möchte ihm zudem für seine freundliche Unterstützung, dem Bereitstellen eines atemberaubend schönen Luftbilds ehemaliger Reisfelder in South Carolina und des Übermittelns eines an ihn adressierten Briefs von Judith Carney danken: David S. Soliday, E-Mail an Jasmin Joppich, 21. 8. 2018. 
direkten Vergleich der Felder ermöglicht. ${ }^{112}$ Derzeit werden der Kultur- und Technologietransfer von Westafrika insbesondere nach South Carolina von Fachleuten aus der historischen, der geographischen, der anthropologischen, der linguistischen und der folkloristischen Disziplin untersucht. ${ }^{113}$ Solidays umfangreiches Projektvorhaben könnte den historischen Kultur-, Technologie- und Wissenstransfer auf Basis von Luftaufnahmen untermauern und somit eine wichtige Grundlage für die weitere Forschung bieten.

\section{Literatur}

Agha, Andrew, Standing the test of time. Embankment investigations, their implications for African technology transfer and effect on African American archaeology in South Carolina, in: Atlantic Studies 12 (2015), Nr. 3, S. 336-354, [https://www.tandfonline.com/doi/full/10.1080/14788810.2015.1062324], eingesehen 15. 8. 2018.

Barnes, Jodi A./Dobrasko, Rebekah, South Carolina's Tidal Rice Fields. Consultation, Collaboration, and Cultural Landscapes, in: International Journal of Cultural Property 21 (2014), Nr. 4, S. 423-444.

Carnegie Mellon University, Edda L. Fields-Black, 2019, [https://www.cmu.edu/dietrich/ history/people/faculty/fields-black.html], eingesehen 7. 2. 2019.

Carney, Judith, Black Rice. The African Origins of Rice Cultivation in the Americas, Cambridge 2001.

Dies., From Hands to Tutors. African Expertise in the South Carolina Rice Economy, in: Agricultural History 67 (1993), Nr. 3, S. 1-30, [https://www.jstor.org/stable/3744227], eingesehen 27. 7. 2018.

Dies., Landscapes of Technology Transfer. Rice Cultivation and African Continuities, in: Technology and Culture 37 (1996), Nr. 1, S. 5-35, [https://www.jstor.org/stable/3107200], eingesehen 27. 7. 2018.

Dies., Out of Africa. Colonial Rice History in the Black Atlantic, in: Sandra G. Harding (Hrsg.), The postcolonial science and technology studies reader, Durham, NC 2011, S. $140-149$.

Coclanis, Peter A., White Rice. The Midwestern Origins of the Modern Rice Industry in the United States, in: Dagmar Schaefer/Edda L. Fields-Black u. a. (Hrsg.), Rice. Global networks and new histories, Cambridge 2015, S. 291-317.

Eltis, David/Morgan, Philip/Richardson, David, Agency and Diaspora in Atlantic History. Reassessing the African Contribution to Rice Cultivationin the Americas, in: The American Historical Review 112 (2007), Nr. 5, S. 1329-1358, [https://www.jstor.org/stable/40007098], eingesehen 27. 7. 2018.

112 An dieser Stelle sei Judith Carney herzlich gedankt, die es mir freundlicherweise gestattet, den Inhalt ihres persönlichen Briefs an David S. Soliday in der vorliegenden Abhandlung zusammenzufassen: Judith Carney, E-Mail an Jasmin Joppich, 11. 2. 2019.

113 Judith Carney, Brief an David S. Soliday, Los Angeles, 24. 1. 2012. 
Fields-Black, Edda L., Atlantic rice and rice farmers. Rising from debate, engaging new sources, methods, and modes of inquiry, and asking new questions, in: Atlantic Studies 12 (2015), Nr. 3, S. 276-295, [https://www.tandfonline.com/doi/full/10.1080/14788810. 2015.1068080], eingesehen 15. 8. 2018.

Dies., Deep Roots. Rice farmers in West Africa and the African diaspora, Bloomington 2008.

Food and Agriculture Organization of the United Nations, FAO Rice Market Monitor (RMM) (1), 2018, [http://www.fao.org/3//9243EN/i9243en.pdf], eingesehen 7. 2. 2019.

Hawthorne, Walter, The Cultural Meaning of Work. The "Black Rice Debate" Reconsidered, in: Schaefer/Fields-Black u. a. (Hrsg.), Rice, S. 279-290.

Kelly, Kenneth G., Rice and its consequences in the greater "Atlantic" world, in: Atlantic Studies 12 (2015), Nr. 3, S. 273-275, [https://doi.org/10.1080/14788810.2015.1062699], eingesehen 7. 2. 2019.

Littlefield, Daniel, Rice and Slaves. Ethnicity and the Slave Trade in Colonial South Carolina, Baton Rouge, London 1981.

Mercer, Edward, Colonial Carolina's Natural Resources, 2018, 1. 9. 2018, [https://sciencing.com/colonial-carolinas-natural-resources-8329601.html], eingesehen 12. 11. 2018.

Moore, Alexander, Rezension zu: Selling a New World. Two Colonial South Carolina Promotional Pamphlets by Thomas Nairne and John Norris by Thomas Nairne, John Norris and Jack P. Greene, in: The South Carolina Historical Magazine 90 (1989), Nr. 4, S. 324426, [https://www.jstor.org/stable/27568096], eingesehen 7. 2. 2019.

Nash, R. C., South Carolina and the Atlantic Economy in the Late Seventeenth and Eighteenth Centuries, in: The Economic History Review 45 (1992), Nr. 4, S. 677-702, [https:// www.jstor.org/stable/2597414], eingesehen 7. 2. 2019.

Siebenmorgen, Terry J., Rice Production, Consumption and Milling Capacity in the United States of America, in: Joachim Sontag (Hrsg.), Rice processing. The comprehensive guide to global technology and innovative products, Clenze 2014, S. 206-213.

Smith, Hayden R., Reserving Water. Environmental and Technological Relationships with Colonial South Carolina Inland Rice Plantations, in: Schaefer/Fields-Black u. a. (Hrsg.), Rice, S. 189-211.

Sontag, Joachim, Introduction, in: Sontag (Hrsg.), Rice processing, S. 3-4.

South Carolina Department of Natural Resources, 2019, [http://www.dnr.sc.gov/], eingesehen 7. 2. 2019.

Stöver, Bernd, United States of America. Geschichte und Kultur. Von der ersten Kolonie bis zur Gegenwart, München $2013^{2}$.

The National Museum of American History, Rice Fanner Basket, Mt. Pleasant, South Carolina, 1970s, O. D., [http://americanhistory.si.edu/collections/search/object/ nmah_659983], eingesehen 12.11.2018. 
U.S. Fish and Wildlife Service, 21.3.2019, [https://www.fws.gov/], eingesehen 7. 2. 2019. UNC College of Arts \& Sciences, Peter A. Coclanis, o. D., [http://history.unc.edu/people/ faculty/peter-coclanis/], eingesehen 27. 7. 2018.

Vanacichit, Apichart, Facts about Rice, in: Sontag (Hrsg.), Rice processing, S. 11-24.

Wang, Muhua/Yu, Yeisoo u. a., The genome sequence of African rice (Oryza glaberrima) and evidence for independent domestication, in: Nature Genetics 46 (2014), Nr. 9, S. 982-991, [https://www.nature.com/articles/ng.3044.pdf], eingesehen 7. 2. 2019.

\section{Quellen}

Carney, Judith, Brief an David S. Soliday, Los Angeles, 24. 1. 2012.

Dies., E-Mail an Jasmin Joppich, 11. 2. 2019.

Collinson, P., Of the Introduction of Rice and Tar in Our Colonies, in: Gentleman's Magazine 36 (1766), S. 278-280, [https://babel.hathitrust.org/cgi/pt?id=mdp.390150183953 53;view=1 up;seq=307], eingesehen 12. 8. 2018.

Richards, Thomas Addison, The Rice Lands of the South, in: Harper's New Monthly Magazine 19 (1859), Nr. 114, S. 721-738, [https://archive.org/details/harpersnew19harper], eingesehen 27. 7. 2018.

Soliday, David S., E-Mail an Jasmin Joppich, 21. 8. 2018.

Ders., o. D., [http://www.davidsoliday.com/rice/], eingesehen 23. 8. 2018.

Waud, Alfred Rudolph, Sketches on a Rice Plantation, in: Harper's Magazine. A Journal of Civilization (1867), Nr. 523, S. 5-6, [https://archive.org/stream/ harpersweeklyv11 bonn\#page/4], eingesehen 22. 8. 2018.

Jasmin Joppich ist Studentin des Unterrichtsfachs Geschichte, Sozialkunde und Politische Bildung an der Universität Innsbruck. jasmin.joppich@student.uibk.ac.at

\section{Zitation dieses Beitrages}

Jasmin Joppich, African Rice Cultivation. Wissens- und Technologietransfer von westafrikanischem Reisanbau nach South Carolina, USA, in: historia.scribere 11 (2019), S. 43-62, [http://historia.scribere.at], eingesehen 17.6.2019 (=aktuelles Datum).

(C) Creative Commons Licences 3.0 Österreich unter Wahrung der Urheberrechte der Autorlnnen. 